Johanna LAAKSO (Vienna)

\title{
ELDIA - a Finno-Ugric minority research project approaching its goal
}

\section{A Finno-Ugric view on European language diversity}

The last few years have seen two major international projects representing a new type of research on the Uralic languages. The Ob-Ugric EuroBABEL project ${ }^{1}$, an international enterprise funded by the European Science Foundation and its national partner agencies, operated from 2009 to 2012, while ELDIA, a research project funded by the 7th Framework Programme of the EU, officially started in March 2010 and ended in September 2013; the last publications of this project are now being prepared. The themes and scholarly approaches of these projects were not completely novel, but what was new was the use of international research funding and cooperation, instead of traditional research structures based on one institution, region or country. These two projects - especially ELDIA, funded by the EU with a total sum of $€ 2.669 .266$ - probably represent the largest international investment until now in the research of Uralic languages.

ELDIA (European Language Diversity for All) set out to investigate the state and conditions of language diversity in Europe by way of diverse Finno-Ugric minorities from Norway to Slovenia. The idea was to utilise the existing research infrastructure and networks for a novel comparative approach which would produce results generalisable for other minority languages and multilingual speaker communities as well. The Finno-Ugric minorities cover practically the whole spectrum of multilingual situations, from very small and extremely endangered languages to numerically strong and relatively stable speaker communities, from well-established, cultivated and standardised languages to language varieties which are only in the process of being standardised and literacised, from indigenous and autochthonous minorities to relatively recent migrant groups - and groups which challenge the dichotomy between autochthonous ("traditional", "regional") and migrant communities. (The Finns in Sweden, for instance, are mostly 1st-to-3rd-generation immigrants, but they also represent a migration pattern and a Finnish presence in Sweden which has existed throughout the documented history of Sweden and Finland.)

By departing from the reality of Finno-Ugric minority languages and including both traditional minorities and migrant groups, ELDIA offered a view complementary to traditional minority language research, which in Europe typically concentrates on a handful of well-defined and well-researched traditional Western European minorities (such as the Celtic minorities, Catalan, Basque, and Frisian). Moreover,

1. Project homepage: $<$ http://www.babel.gwi.uni-muenchen.de $>$. 
the main goal of ELDIA was not minority research as such but research into living multilingualism in its various forms, which may include both acquired multilingualism (for instance, the learning of major vehicular languages such as English) and the "natural" multilingualism of minority groups (for instance, growing up speaking both Hungarian and German) - one of the basic tenets of the project was that there is no sharp distinction between these two.

\section{International and interdisciplinary work}

The ELDIA project was run by an international and interdisciplinary consortium, consisting of senior researchers from the universities of Helsinki, Oulu, Mainz, Maribor, Stockholm (until March 2011), Tartu and Vienna and the Åland Islands Peace Institute, and including experts on linguistics, law, sociology (social anthropology, media studies) and statistics. The coordination was centered at the Johannes Gutenberg University in Mainz, with Professor Anneli Sarhimaa, the instigator of the entire project, as the coordinator-in-chief.

Most of the project's work was organised in subsequent work packages building on each other. The idea was to conduct case studies based on the same research design with 14 different minorities: North Sámi and Kven in Norway, Meänkieli speakers and Finns in Sweden, Estonian migrants in Finland and Germany, Karelian speakers in Finland and Russia, Veps speakers in Russia, Võro speakers in Estonia, Seto speakers in Estonia and Russia, and Hungarians in Austria and Slovenia. Of these studies, one could not be realised and another was left unfinished: the University of Stockholm, responsible for the case study on Finns in Sweden, left the project in 2011, while fieldwork on the Seto in Russia, due to the small numbers of the target population and the lack of supporting infrastructure, turned out to be practically impossible.

The work began with so-called context analyses summarising the state-of-art in research and the available knowledge about each target community. These analyses, written according to a centrally planned template, were used for the planning of the fieldwork: the questionnaire survey and interviews, both of which targeted both the minority group at issue and a control group representing the majority population. The questionnaire data and the interviews, in turn, were analysed, again on the basis of a centrally planned research design. At the same time, two international research teams worked on their own analyses, mainly based on desk research: a law team analysed the legal and institutional frameworks in each country or region at issue, and a media-sociological team investigated minority and majority media discourses and their roles in representing the minority group at issue, reflecting and reproducing power relations.

The empirical work, involving the efforts of dozens of junior researchers and research assistants, was even more challenging than had been anticipated. In particular, problems arose with the University of Stockholm, the partner in charge of the 
empirical research design, and in the end Stockholm had to leave the project. These problems delayed the questionnaire survey and all the subsequent work phases, and extracting data from the questionnaires - without knowing what the plans behind the questionnaire design had been - constituted a real challenge for the data analysis team. The technical preparation of the questionnaires, which had to be translated into eighteen different minority and majority languages, and processing them into machine-readable form was already a major effort. Despite practical difficulties and challenging conditions - as in the fieldwork among the Karelians and Veps in Russia during the coldest time of winter in early 2011 - the work was finished, thanks to the commitment of the research teams. The results of the data analyses were gathered into case-specific reports, based again on a centrally planned template.

\section{Assessing language maintenance: EuLaViBar}

One of the main goals of the project was to create a tool for assessing language endangerment: a barometer which can be used to measure the state of maintenance of any potentially endangered language and to identify those areas in language maintenance which are especially in need of support. The European Language Vitality Barometer (EuLaViBar) can be visualised with a polar diagram (radar chart), divided into four quadrants, each of which consists of 3 or 4 sectors or "dimensions" (language use and interaction, education, legislation, and media). The quadrants represent the so-called focus areas, three of them based on the three basic conditions for language maintenance as identified by François Grin and his associates: Capacity, Opportunity, and Desire. The fourth focus area, Language products, was inspired by Miquel Strubell's "Catherine Wheel" model, which describes the revitalisation of a language in terms of demand and supply of products and services in the language at issue.

For each ELDIA case study, the results from selected questions of the questionnaires were processed into numeric values on a simple scale from 0 (severely and critically endangered) to 4 (maintained at the moment). Each of these values reflects the maintenance of the language at issue in a certain focus area and dimension of language use, that is, in a certain sector of the diagram. The resulting EuLaViBar diagrams, together with explanations and comments, have been published in the respective case-specific reports. On the basis of these experiences, an amended version of the questionnaire and a EuLaViBar Toolkit have been published on the project website; they can be used and developed for further investigations of language maintenance ("vitality") or endangerment.

Creating scales for the assessment of language endangerment or (ethno)linguistic vitality is not complicated per se, and many models and tools already exist many of them inspired by the most famous one, the GIDS (Graded Intergenerational Disruption Scale) by Joshua Fishman. The EuLaViBar model, however, differs from most of these in that it is based on large amounts of empirical data. Moreover, the 
model describes the maintenance of a language as a sum of different factors, and it is possible to break the barometer values down into their constitutive parts for a more fine-grained analysis of the situation.

\section{Results and policy implications}

The most obvious and critical result of the ELDIA analyses was that all the minority languages investigated are endangered to various extents. In particular, it was interesting to notice that the three minority languages with the poorest overall scores - Kven, Meänkieli, and Karelian in Finland - are all spoken in the Nordic countries, which otherwise are known for their high rankings in numerous international comparisons of human development, democracy and human rights. Obviously, even strong traditions of democracy and egalitarianism cannot automatically guarantee the maintenance of minority languages, nor does mere non-discrimination suffice to compensate for the consequences of earlier assimilatory policies.

Despite the fact that in all countries under study, language and/or minority laws have been passed or amended recently, the legal and institutional support was perceived as inadequate, and the respondents were often ignorant, sceptical or indifferent concerning their linguistic rights. Furthermore, one of the most interesting overall findings of the law team was that nowhere is multilingualism in itself endorsed or protected by law: what is protected is, in the best case, the right of a certain group to use a certain language in a certain area or region, and language policies may still be based on the outdated idea of monolingual and clearly delimitable ethnic communities.

As for media and language products, the analyses of media discourses showed that both minority and majority media often avoid critically discussing really relevant matters such as the effects of language policies or the problems of language revitalisation. Rather, they focus on "harmless" issues of culture and tradition and may reproduce the "extinction narrative", portraying the minority and its culture as part of the past. The language products and services available were inadequate and insufficient throughout the cases examined. In particular, the education systems, even if they offer teaching in the minority language, often fail to produce real functional bilingualism - or do not even define functional bilingualism as their goal.

As the most important policy recommendation, the need for strategies supporting sustainable multilingualism emerges. Considering that all speaker communities under study both expressed their wish to maintain their heritage language and acknowledged the importance of knowing the local majority language and other important vehicular languages, supporting functional multilingualism on individual and societal level is the only viable solution if citizens' wishes and their linguistic rights are to be respected. 


\section{Publications and data}

Most of the products of ELDIA have appeared or will soon appear in the electronic open-access publication series Studies in European Language Diversity (earlier: Working Papers in European Language Diversity). These include the abridged versions of the context analyses which were prepared in the first work phase of the project, the country-specific reports of the law team, and the case-specific reports. The case-specific reports comprise or summarise the results of the context analysis, the questionnaire survey, the interviews, and the law and media analyses, and also include further policy recommendations for each case. Furthermore, of each case-specific report abridged translations in the minority and majority language at issue will be published. All these publications are or will soon be available on the project website $(<w w w$.eldia-project.org $>$ ) or directly at $<$ http://phaidra.univie.ac.at/o:80726>.

The EuLaViBar Toolkit, available on the project website, can be used for further assessments of language maintenance, and all interested experts are invited to adapt and to develop it further.

The fieldwork data, including, among other things, approximately 190 hours of interview recordings with transcriptions, will be stored in the ELDIA-DATA database at the University of Mainz. The database can be used for research purposes; requests should be submitted to the ELDIA consortium via < sarhimaa@uni-mainz.de>. 